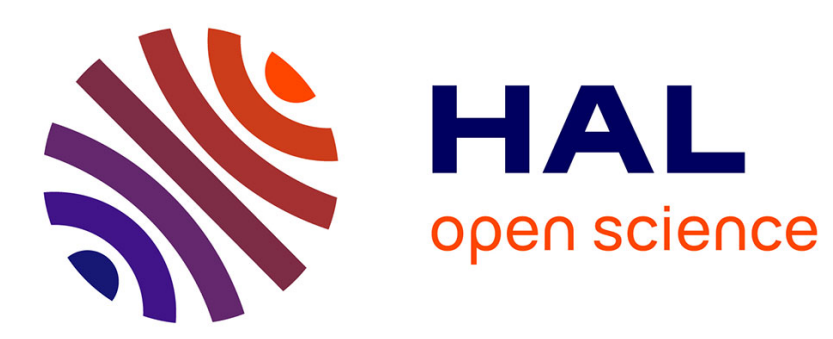

\title{
Adaptive expectations and cobweb phenomena: does heterogeneity matter?
}

Domenico Colucci, Vincenzo Valori

\section{To cite this version:}

Domenico Colucci, Vincenzo Valori. Adaptive expectations and cobweb phenomena: does heterogeneity matter?. Journal of Economic Dynamics and Control, 2011, 10.1016/j.jedc.2011.03.008 . hal-00828981

\section{HAL Id: hal-00828981 \\ https://hal.science/hal-00828981}

Submitted on 1 Jun 2013

HAL is a multi-disciplinary open access archive for the deposit and dissemination of scientific research documents, whether they are published or not. The documents may come from teaching and research institutions in France or abroad, or from public or private research centers.
L'archive ouverte pluridisciplinaire HAL, est destinée au dépôt et à la diffusion de documents scientifiques de niveau recherche, publiés ou non, émanant des établissements d'enseignement et de recherche français ou étrangers, des laboratoires publics ou privés. 


\section{Author's Accepted Manuscript}

Adaptive expectations and cobweb phenomena: does heterogeneity matter?

Domenico Colucci, Vincenzo Valori

PII: S0165-1889(11)00053-4

DOI: doi:10.1016/j.jedc.2011.03.008

Reference: DYNCON 2554

To appear in: $\quad$ Journal of Economic Dynamics

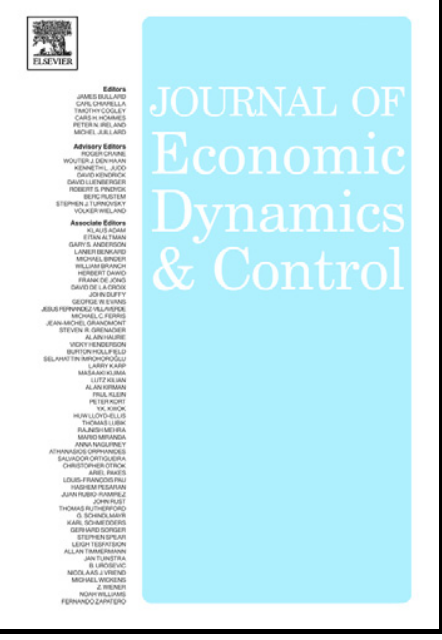

www.elsevier.com/locate/jedc \& Control

Received date: $\quad 31$ March 2009

Revised date: $\quad 10$ January 2011

Accepted date: 9 March 2011

Cite this article as: Domenico Colucci and Vincenzo Valori, Adaptive expectations and cobweb phenomena: does heterogeneity matter?, Journal of Economic Dynamics \& Control, doi:10.1016/j.jedc.2011.03.008

This is a PDF file of an unedited manuscript that has been accepted for publication. As a service to our customers we are providing this early version of the manuscript. The manuscript will undergo copyediting, typesetting, and review of the resulting galley proof before it is published in its final citable form. Please note that during the production process errors may be discovered which could affect the content, and all legal disclaimers that apply to the journal pertain. 


\title{
Adaptive expectations and cobweb phenomena: does heterogeneity matter?
}

\author{
Domenico Colucci $^{\mathrm{a}}$, Vincenzo Valori ${ }^{\mathrm{a}, *}$ \\ ${ }^{a}$ Università di Firenze, Via C. Lombroso 6/17, 50134 Firenze, Italy.
}

\begin{abstract}
This paper studies a cobweb-type commodity market characterised by a strictly monotone demand and supply, in which $n$ types of firms operate. Types differ in a key parameter governing price expectations which are supposed to be adaptive. The unique steady state of the resulting economic dynamics is characterized in terms of stability and the impact of the number of firms types is studied: to this end the notions of structural and behavioural degree of instability, which are introduced in the paper, prove to be crucial in determining whether stability or instability prevail. The case of market integration is also considered and conditions to have stability (or instability) in terms of the original markets' parameters are given. The baseline structure is extended in two directions. The first extension assumes the point of view of an authority who is uncertain about the firms types. In this case the structural degree of instability determines how heterogeneity affects the probability of ending up with a stable outcome. The second extension consists in endogenizing the choice of predictors through a discrete choice based evolutionary mechanism. In both cases the amount of the heterogeneity and its possible variations play a critical role in shaping the range of possible long-run outcomes of the model.
\end{abstract}

Journal of Economic Literature Classification Numbers: D83; D84; E17; E32

Keywords: heterogeneous expectations, bounded rationality, stability of steady states, market integration, evolutionary dynamics

\section{Introduction and related literature}

The existence of a certain amount of heterogeneity in economic expectations is uncontroversial. Evidence of heterogeneous expectations in inflation forecasts for example (both by professionals and non-professional forecasters) has been presented by Branch [2], Carroll [6] and Mankiw et al. [21]. The extent, the variability and the consequences of such expectations disagreement are an open and interesting research question. The range of applications and models in which such question receives attention includes for

\footnotetext{
*Corresponding author

Email addresses: domenico.colucci@unifi.it (Domenico Colucci), vincenzo.valori@unifi.it (Vincenzo Valori)

Preprint submitted to Elsevier

March 11, 2011
} 
example monetary policy theory and design (as in Honkapohja and Mitra [16]), models of exchange rate dynamics (e.g. Manzan and Westerhoff [22]) and asset pricing (for recent examples see Buraschi and Jiltsov [4], Jouini and Napp [19]). Heterogeneous expectations have also been invoked to explain stylized facts such as the volume of trade exchange (see e.g. Frankel and Froot [13]). Further, for some types of agricultural markets in which biological lags naturally suggest the use of cobweb models, heterogeneous expectations have been detected and estimated, for instance by Chavas [7]. A rather comprehensive survey on these studies can be found in Hommes [18].

The present work is part of a thread of literature dealing with the cobweb model under bounded rationality which goes back to studies by Nerlove [24] and Carlson [5] a few decades ago. More recently Hommes [17] investigated a homogeneous cobweb model with adaptive expectations, a linear demand curve and a nonlinear supply curve and showed that adaptive expectations stabilize the amplitude of price fluctuations, but at the same time these smaller price fluctuations become more complicated, even chaotic.

In essence, this paper studies the conditions under which coordination or disagreement of beliefs among individuals of limited rationality emerge and the impact of a moderate degree of expectations' heterogeneity on dynamic stability. In particular we consider the problem of characterizing dynamic stability of equilibria in a cobweb model in which $n$ types of firms use adaptive expectations with specific gain parameters. The heterogeneity we take into account is indeed moderate because it is limited to a key parameter governing expectations which are otherwise all drawn from the same expectations mechanism. It turns out that in our model two sources of (potential) instability can be identified: a structural source, linked to the market's fundamentals (such as the shape of demand and supply curves) and a behavioural source, embedded in the profile of expectations characterizing the suppliers. A necessary and sufficient condition for local stability involving these factors is demonstrated. Such condition implies no particular restriction on individual firms, but only on the entire set of firms as a whole. For the asymptotically stable configurations we characterize the situations in which convergence occurs monotonically and those in which the steady state is approached through oscillations giving rise to the traditional cobweb phenomena.

The structural and behavioural sources of instability also show up as we study the effects of modifying the number of firms types. A situation in which this can happen without altering the remaining structure of the market may arise for example as producers form a (or walk out from) a consortium so that their heterogeneous expectations cease to (or start to) affect the market directly. On the contrary the heterogeneity changes along with other important structural aspects when two markets that were previously separated are joined into one bigger marketplace, for example as a result of trading agreements between states, or of the introduction of new technology allowing such a change.

Motivated by the difficulty to actually observe expectations, whereas it is easier to measure some structural features of a given market, such as the relevant demand and supply price elasticities, we take the perspective of an observer (e.g. a policy maker) who knows the structural parameters of the market but is uncertain about the behavioural ones. A similar perspective was applied by Colucci and Valori [9] to an asset-pricing model. The existence of a probability distribution of the behavioural parameters defining types is assumed and probabilities of convergence conditional on the structural source of instability are worked out. A form of polarization of convergence probabilities induced 
by increasing the heterogeneity is documented. When the number of types gets large, stability is almost certain for levels of the structural parameters up to a certain threshold, while the system is almost certainly unstable past the threshold.

Finally the model is extended to allow the possibility for the firms to endogenously switch between different values of the adaptive parameter on the basis of the past payoffs attached to each such value (whether directly observed or ex-post calculated), using an evolutionary mechanism of discrete choice among predictors. The upshot is that, while the variety of possible behaviors increases thanks to the stochastic elements introduced, forms of polarization similar to those encountered in the model without switching emerge, thus suggesting a certain robustness of these features connected to variations in the degree of expectatations heterogeneity.

Brock and Hommes [3] introduced the possibility for firms in the cobweb to choose between costly rational expectations and naive expectations, using a stochastic discrete choice model and documenting complex dynamics within an adaptively rational equilibrium (the so-called rational route to randomness). Later, Goeree and Hommes [14] generalized this model to nonlinear (monotonic) demand and supply, while Branch [1] introduced a kind of adaptive expectations (or rather a fading memory mechanism) within the menu of available predictors. Similarly, Lasselle et al. [20] consider the cobweb model with costly rational versus free adaptive expectations. With respect to these papers the present work stands in a somewhat slant position, in the sense that while our basic setup can be considered a special case of the Brock and Hommes [3] cobweb model, the focus here is different, given that, even when switching is allowed for, there is not a globally "best" predictor to choose. At the same time our analysis places no particular restrictions on supply and demand and number of adaptive types, attaining hitherto undescribed results about the effect of multy-type adaptive expectations firms within a general cobweb model.

Other works in which the role of producers' heterogeneity within the cobweb model is inquired include Chiarella et al. [8], where the heterogeneity is in the lag lengths and memory parameters applied to past prices as well as in risk aversion coefficients and Onozaki et al. [25] where producers are either 'naive optimizers' or 'cautious adapters': in both these papers heterogeneity can result in dramatic consequences with respect to the relevant representative agent benchmark. Negroni [23] investigates a two-agents problem with adaptive expectations which is akin to this paper's but for the assumed asymmetry in the roles of the agents which is absent here. A closely related feature also shows up in the paper by Evans and Guesnerie [11], who name it "structural heterogeneity" and show its potential role of destabilising force when coupled with different beliefs.

The paper is organised as follows: Section 2 introduces the model and states a couple of results which are then used throughout the paper. The issue of understanding the specific role of the number of firms in shaping stability is addressed in Section 3. Section 4 deals with the probability of convergence when there is uncertainty about the firms' behavioural characteristics. Section 5 considers the endogenous selection of adaptive parameters through a discrete choice model and reports results of several simulation exercises. All the proofs are contained in the Appendix. 


\section{The Model}

Consider a cobweb-type commodity market in which each firm, that come in $n$ different types, needs to allow for a production lag and so choose optimal supplied quantities conditioned on the forecasted future price $p_{i}^{e}$, which characterizes firms of type $i=1, \ldots, n$. The total optimal supply for firms of type $i$ is proportional to their aggregate size, $\psi_{i}>0$, hence

$$
S_{i}\left(p_{i}^{e}\right)=\psi_{i} s\left(p_{i}^{e}\right)
$$

where $s\left(p_{i}^{e}\right)$ solves the expected profit maximization problem

$$
\max _{x} p_{i}^{e} x-c(x)
$$

given that $c(x)$ is the (common) cost function which describes the available production technology on the market. ${ }^{1}$ The demand is a function of the current price, $D(p)$. We assume a strictly increasing supply ${ }^{2}$ and a strictly decreasing demand, which are smooth and intersecting at a point $p^{*}$. By defining $\Psi=\sum_{i} \psi_{i}$ as the aggregate production scale factor, $S(\cdot)=\Psi s(\cdot)$ and $\phi_{i}=\frac{\psi_{i}}{\Psi}$ as the market share of firms of type $i$ (with $\left.\sum_{i=1}^{n} \phi_{i}=1\right)$ the aggregate supply becomes

$$
\hat{S}\left(p_{t, 1}^{e}, \ldots, p_{t, n}^{e}\right)=\Psi \sum_{i=1}^{n} \phi_{i} s\left(p_{t, i}^{e}\right)=\sum_{i=1}^{n} \phi_{i} S\left(p_{t, i}^{e}\right) .
$$

Market clearing requires that $D\left(p_{t}\right)=\sum_{i=1}^{n} \phi_{i} S\left(p_{t, i}^{e}\right)$ which, because demand is strictly increasing, can be written explicitly as

$$
p_{t}=D^{-1}\left(\sum_{i=1}^{n} \phi_{i} S\left(p_{t, i}^{e}\right)\right) \equiv F\left(p_{t, 1}^{e}, \ldots, p_{t, n}^{e}\right)
$$

The way we model the relative sizes of the different types of producers on the market is quite standard (see e.g. Onozaki et al. [25], Chiarella and He [8]), while the absolute sizes $\psi_{i}$ will only play a role later (see Section 3$) .^{3}$

We close the model assuming that expectations are adaptive with gains that differ across different types of firms $p_{t+1, i}^{e}=p_{t, i}^{e}+\alpha_{i}\left(p_{t}-p_{t, i}^{e}\right), i=1, \ldots, n$. Summing up, the evolution of the system can be described by the following system of difference equations

$$
\left\{\begin{array}{c}
p_{t+1,1}^{e}=p_{t, 1}^{e}+\alpha_{1}\left(F\left(p_{t, 1}^{e}, \ldots, p_{t, n}^{e}\right)-p_{t, 1}^{e}\right) \\
\ldots \\
p_{t+1, n}^{e}=p_{t, n}^{e}+\alpha_{n}\left(F\left(p_{t, 1}^{e}, \ldots, p_{t, n}^{e}\right)-p_{t, n}^{e}\right)
\end{array}\right.
$$

\footnotetext{
${ }^{1}$ Notice that the ex-post profit for the firm might be negative. Here we are implicitly assuming a nobankruptcy condition. The issue of market exit and profitability has been taken seriously and examined by e.g. Commendatore and Currie [10].

${ }^{2}$ This can be easily justified assuming a strictly convex cost function in (1).

${ }^{3}$ This setup is compatible with a continuum of firms as in Brock and Hommes [3] and related literature as well as with a finite number of firms, large enough to maintain the assumption of competitive market. The paper can be read within both interpretations, with the exception of Section 5 where we explicitly posit a finite number of firms.
} 
The above assumptions on the monotonicity of supply and demand guarantee that there will be a unique steady state for the system (3), corresponding to the supply-demand equilibrium price $p^{*}$. Notice that, in spite of the simplicity of adaptive expectations, the number of different firms determines the dimension of the dynamical system (3): this is a distinguishing feature of the model. Assuming for example that the $n$ types of firms use $A R(p)$ forecasting models (with lags up to a given $p$ ) would make the dimension of the system independent of $n$ : so a large number of types would not, as it does here, complicate the tractability of the model.

\subsection{Special case: mono-type firms}

It is useful to see what happens if there is only one firm type (see Hommes [17]). In this case the price equation (2) reduces to $p_{t}=D^{-1}\left(\Psi s\left(p_{t}^{e}\right)\right)=D^{-1}\left(S\left(p_{t}^{e}\right)\right)$ so the system evolves according to

$$
p_{t+1}^{e}=p_{t}^{e}+\alpha\left(D^{-1}\left(S\left(p_{t}^{e}\right)\right)-p_{t}^{e}\right)
$$

and the stability condition is $-1<1-\alpha+\alpha \frac{S^{\prime}\left(p^{*}\right)}{D^{\prime}\left(p^{*}\right)}<1$ which, defining $\delta=-\frac{S^{\prime}\left(p^{*}\right)}{D^{\prime}\left(p^{*}\right)}$, we can write as $-1<1-\alpha-\alpha \delta<1$. Using the definition $\beta=\frac{\alpha}{2-\alpha}$ and the fact $\delta>0$, stability requires that

$$
\delta \beta<1
$$

As it turns out, the two parameters ${ }^{4} \delta, \beta$ play a key role throughout the paper. Label $\delta$ the structural degree of instability. Notice that as $\delta$ approaches 1 condition (4) is automatically satisfied for any choice of $\alpha \in(0,1)$, and if $\delta \leq 1$ stability is always warranted under adaptive expectations. Therefore we assume $\delta>1$. The parameter $\beta$ will be called the behavioural degree of instability.

\subsection{General case: $n$ types}

We now turn to the issue of how stability for the model in its general form with $n$ types relates to (behavioural) characteristics of the individual firms and to the market's exogenous structure (as given by the demand and supply functions). To this end notice preliminarily that

$$
\left.\frac{\partial F\left(p_{t, 1}^{e}, \ldots, p_{t, n}^{e}\right)}{\partial p_{t, i}^{e}}\right|_{p_{t, 1}^{e}=\cdots=p_{t, n}^{e}=p^{*}}=\phi_{i} \frac{S^{\prime}\left(p^{*}\right)}{D^{\prime}\left(p^{*}\right)}=-\phi_{i} \delta
$$

so the Jacobian matrix of the system (3) evaluated at $p^{*}$ is:

$$
J_{n}=\left(\begin{array}{cccc}
1-\alpha_{1}\left(\phi_{1} \delta+1\right) & -\alpha_{1} \phi_{2} \delta & \cdots & -\alpha_{1} \phi_{n} \delta \\
-\alpha_{2} \phi_{1} \delta & 1-\alpha_{2}\left(\phi_{2} \delta+1\right) & \cdots & -\alpha_{2} \phi_{n} \delta \\
\ldots & \ldots & \ddots & \ldots \\
-\alpha_{n} \phi_{1} \delta & -\alpha_{n} \phi_{2} \delta & \cdots & 1-\alpha_{n}\left(\phi_{n} \delta+1\right)
\end{array}\right)
$$

\footnotetext{
${ }^{4}$ Notice that in turn $\delta$ depends on a third parameter, namely $\Psi$, the overall dimension of the economy. This is relevant only when market integration is discussed.
} 
It seems fairly intuitive that if the conditions for individual stability derived above in (4) are met (or violated) for each group of firms then stability (or instability) will prevail on the market. Indeed this needs not be the case in general, as Franke and Nesemann [12] have shown in a specific case in which two "unstable" learning rules offset each other bringing about a stable outcome. In our context, given the limited degree of freedom in firms behaviour, such phenomenon is not possible and stability (or instability) at the individual level suffice for stability (instability) with many types. Notice further that the market can be stable even though stability conditions (4) do not hold for all types: consider, for example, the case of a market with two groups of equal size having $\delta=2$, $\beta_{1}=\frac{1}{4}$ and $\beta_{2}=\frac{2}{3}$. Because $\delta \beta_{1}<1<\delta \beta_{2}$, condition (4) entails that, in isolation, the first group implies stability whereas the second implies instability, but overall the market is stable (direct calculation or Proposition 2 show that such is the case). So it is interesting to establish conditions by which stability is produced when $n$ types of firms supply the good. The following preliminary result is required.

Lemma 1. Consider a matrix

$$
M=\left(\begin{array}{cccc}
a_{1} c_{1}+b_{1} & a_{1} c_{2} & \cdots & a_{1} c_{n} \\
a_{2} c_{1} & a_{2} c_{2}+b_{2} & \cdots & a_{2} c_{n} \\
\vdots & \vdots & \ddots & \vdots \\
a_{n} c_{1} & a_{n} c_{2} & \cdots & a_{n} c_{n}+b_{n}
\end{array}\right)
$$

with $a_{i}<0, b_{i}, c_{i}>0$ for all $i$. Then

i) $\operatorname{det} M=\prod_{i=1}^{n} b_{i}+\sum_{i=1}^{n} c_{i} a_{i} \prod_{j \neq i} b_{j}$

ii) $M$ has real eigenvalues

iii) $(n-1)$ eigenvalues of $M$ belong to the interval $\left[\min _{i}\left\{b_{i}\right\}, \max _{i}\left\{b_{i}\right\}\right]$ and for the smallest eigenvalue of $M, \lambda_{\min }$, it is $\lambda_{\min }<\min _{i}\left\{b_{i}\right\}$

iv) $\lambda_{\min }$ is greater than -1 if and only if the characteristic polynomial, $P(\lambda)$, is positive at $\lambda=-1$.

Notice that the Jacobian (5) is a particular specification of matrix (6) with $a_{i}=$ $-\alpha_{i}, b_{i}=1-\alpha_{i}, c_{i}=\phi_{i} \delta$. Therefore, as a consequence of part ii) and iii) of the above Lemma applied to (5), the steady state of system (3) can loose (acquire) stability only through a Period-doubling bifurcation. When the eigenvalues of (5) are all non-negative the local convergence of expectations, quantities and price to their steady state value is monotone: in that case, a perturbation of the model's parameters does not result in a qualitative change of the dynamics around the steady state.

Let us now turn to the stability properties of the steady state of the market dynamics. Define the market degree of behavioural instability for the $n$ heterogeneous firms types case as $\bar{\beta}_{n}=\sum_{i=1}^{n} \phi_{i} \beta_{i}=\sum_{i=1}^{n} \phi_{i} \frac{\alpha_{i}}{2-\alpha_{i}}$. Perhaps surprisingly, stability can be characterised in terms of $\bar{\beta}_{n}$ and $\delta$ in the same way as in the homogeneous case.

Proposition 2. The steady state of the system (3) is locally stable and hyperbolic (i.e. with eigenvalues strictly inside the unit circle) if and only if $\delta \bar{\beta}_{n}<1$.

The result says that, in order to have stability, the multiplicative combination of structural and behavioural instability must not exceed one. So this establishes a threshold 
for the aggregate sources of instability in the market, marking the frontier between the stable and the unstable regimes.

When the steady state is locally stable it is interesting to look for more insights about the path of convergence to the equilibrium. The persistent fluctuating pattern of prices in specific agricultural markets, originally attracted the attention of the economics profession in the 1930s and propelled the development of the cobweb literature. We now identify conditions under which our model implies oscillatory dynamics, in particular along converging paths. Observe that the model allows both for monotone and for nonmonotone convergence, depending on the parameters values.

Proposition 3. The system (3) shows monotonic local convergence to the steady state if and only if $\sum_{i=1}^{n} \phi_{i} \frac{\alpha_{i}}{\left(1-\alpha_{i}\right)}<\frac{1}{\delta}$

Notice that the left hand side of the above inequality tends to 0 with the $\alpha_{i}$, so monotone convergence is always possible independently of the market's structural degree of instability level. Furthermore, as the greatest eigenvalue cannot exceed 1, the robustness of the market stability to parameters perturbations is stronger when convergence is monotone and also, due to Lemma (5) below, it increases when the $\alpha_{i}$ decrease. $^{5}$

\subsection{Heterogenous versus average homogeneous markets}

At this point the reader might raise the following doubt: if the heterogeneity in the market were incorporated within a single representative type of firm, would there be any real loss with respect to the more complex model of Section 2.2? It turns out that the answer is "yes". The following Proposition compares the conditions for stability in the homogeneous market with a single representative type (in the sense that its adaptive gain parameter is equal to the weighted average of parameters of $n$ given types), to those for the heterogeneous market with those $n$ types actually playing directly.

Proposition 4. Consider a market with $n$ types of firms defined by gains $\alpha_{1}, \ldots, \alpha_{n}$ and weights $\phi_{1}, \ldots, \phi_{n}$. Conditions for stability in the heterogeneous market are sufficient but not necessary for the average homogeneous market with suppliers of a single average type having gain $\alpha=\sum_{i=1}^{n} \phi_{i} \alpha_{i}$.

A simple numerical example can illustrate a case in which the heterogeneous market is unstable but its average homogeneous counterpart would be stable. Consider $n=2$, $\phi_{1,2}=1 / 2$ and $\delta=2$. If $\alpha_{1}=1 / 3$ and $\alpha_{2}=9 / 10$ we have

$$
\begin{aligned}
\bar{\beta}_{a v} & =\frac{\frac{1}{2}\left(\frac{1}{3}+\frac{9}{10}\right)}{2-\frac{1}{2}\left(\frac{1}{3}+\frac{9}{10}\right)}=\frac{37}{83}<\frac{1}{2} \Rightarrow \bar{\beta}_{a v} \delta<1 \\
\bar{\beta}_{h e t} & =\frac{1}{2}\left(\frac{\frac{1}{3}}{2-\frac{1}{3}}\right)+\frac{1}{2}\left(\frac{\frac{9}{10}}{2-\frac{9}{10}}\right)=\frac{28}{55}>\frac{1}{2} \Rightarrow \bar{\beta}_{h e t} \delta>1
\end{aligned}
$$

Proposition 4 argues in favour of the idea that heterogeneity matters, from the dynamic stability/instability viewpoint, in that it cannot be safely sterilized by using an

\footnotetext{
${ }^{5}$ On the contrary, the speed of convergence to the steady state is higher when convergence is nonmonotone as we show in the working paper version of this work.
} 
average representation instead of the whole heterogeneous picture. In a sense this result also appears to indicate that, as opposed to the average homogeneous market, heterogeneity implies (or has a potential for) destabilization. More in general, while it is crucial to fix ideas precisely as to what (de)stabilizing heterogeneity means, much depends on the level of structural degree of instability, $\delta$ : Section 4 is specifically devoted to this issue.

\section{Some comparative statics and market integration}

This section deals with the issue of assessing the effect of changes in the amount of behavioural heterogeneity on stability, first in the context of comparative statics (which leaves unchanged the market size) then as a by-product of a process of market integration (whereby two separate markets are merged into a bigger one).

To begin with, suppose there is a change in the number of firms types, $n$. This situation may arise for example as producers form a (or walk out from) a consortium so that their heterogeneous expectations cease to (or start to) affect the market directly. This is a comparative statics exercise in which the menu of available predictors is enriched with a new type, corresponding to a new adaptive gain parameter, $\alpha_{n+1}$ or equivalently to a new behavioural degree of instability, $\beta_{n+1} \cdot{ }^{6}$ We assume that a fraction $1-\rho$ of firms (uniformly across the existing types) switch to using the new parameter, whereas a fraction $\rho$ does not change its behaviour. Letting $\bar{\beta}_{O}$, and $\bar{\beta}_{N}$ the aggregate degree of behavioural instability in the original configuration and in the augmented one

$$
\begin{aligned}
\bar{\beta}_{N} & =\sum_{i=1}^{n} \rho \phi_{i} \frac{\alpha_{i}}{2-\alpha_{i}}+\sum_{i=1}^{n}(1-\rho) \phi_{i} \frac{\alpha_{n+1}}{2-\alpha_{n+1}} \\
& =\rho \bar{\beta}_{O}+(1-\rho) \beta_{n+1}
\end{aligned}
$$

Demand side, supply functions and the equilibrium price are unchanged, so the degree of structural instability, $\delta$ remains the same. The following result is now easy to prove. Due to Proposition 2, stability (or instability) in the enlarged configuration depends on whether $\delta \bar{\beta}_{N}$ is smaller (or larger) than 1 . Trivially $\delta \bar{\beta}_{N}=\delta\left(\rho \bar{\beta}_{O}+(1-\rho) \beta_{n+1}\right) \lessgtr 1$ when $\delta \bar{\beta}_{O}, \delta \beta_{n+1} \lessgtr 1$; hence if the market in its original configuration is stable, it remains so if $\delta \beta_{n+1}<1$. In this context therefore, stability (or instability) persists when a larger span of firms types is allowed for.

If instead $\delta \beta_{n+1}>1$ then the market is stable if and only if $\rho>\frac{\delta \beta_{n+1}-1}{\delta \beta_{n+1}-\delta \beta_{O}}$. This means that when the new type introduced would imply instability if it were the only one, the outcome depends crucially on the fraction $1-\rho$ of firms switching to it: such fraction needs to be under (above) a threshold which depends on the structural and behavioural instability, $\delta, \bar{\beta}_{O}$ and $\beta_{n+1}$, in order for the outcome to be stable (unstable). Again this is a rather intuitive result.

Another question worth addressing is the following: what is the minimum fraction of firms that, by switching to the new parameter, can destabilise the market? Intuitively, the worst that can happen is the introduction of a new type with a behavioural degree

\footnotetext{
${ }^{6}$ The following analysis carries through for any finite number of new types. We stick to this simpler
} case to favour the intuition. 
of instability of 1 (i.e. with $\alpha_{n+1}=1$ ). In that case $\rho<\frac{1-\delta}{\delta \beta_{A}-\delta}$ would make the system unstable. Remark that this argument relies on the idea that switching to $\alpha_{n+1}=1$ is the "worst that can happen". This is indeed the case because the largest eigenvalue is always smaller than 1 while the smallest one is strictly decreasing in $\alpha_{i}$ as we show in the following Lemma.

Lemma 5. If $\lambda_{\max }$ and $\lambda_{\min }$ are the largest and smallest eigenvalues of the Jacobian (5), then $\lambda_{\min }$ strictly decreases with $\alpha_{i}$, for all $i$, while $\lambda_{\max }$ weakly decreases with $\alpha_{i}$, for all $i$ and $\lambda_{\max } \leq 1-\alpha_{\min }$.

Finally we determine a kind of "central value" for the degree of structural instability, $\delta$. To do so, observe that the threshold ratio of firms, $\frac{\delta \beta_{n+1}-1}{\delta \beta_{n+1}-\delta \bar{\beta}_{A}}$, in the polar case of $\bar{\beta}_{O}=0$ and $\beta_{n+1}=1$ simplifies to $1-1 / \delta$. Therefore when $\delta=2$ the threshold fraction separating the stable and the unstable regimes equals exactly $50 \%$ when firms have only either static or myopic expectations (i.e. when the gain $\alpha_{i}$ is either 0 or 1 ). Remarkably, the value of $\delta=2$ can be described as central also for other reasons, as it is shown in Section 4.

\subsection{Market integration}

Suppose two markets that were previously independent are aggregated. This is a situation in which it is natural to expect the resulting increased behavioural heterogeneity to play a role on the observable dynamics of the market.

The equilibrium price in the integrated market will be intermediate between the two original equilibria, thanks to the assumption of monotone demand and supply. The two markets are characterised by the vectors of parameters $\left(\bar{\beta}_{A}, \delta_{A}, \Psi_{A}\right),\left(\bar{\beta}_{B}, \delta_{B}, \Psi_{B}\right)$. Let $p_{A}^{*}<p_{B}^{*}$ be the market equilibria defined by supply and demand. The aggregated market will have $\left(\bar{\beta}, \delta, p^{*}, \Psi\right)$ where $\Psi=\Psi_{A}+\Psi_{B}, \bar{\beta}=\frac{\Psi_{A}}{\Psi} \bar{\beta}_{A}+\frac{\Psi_{B}}{\Psi} \bar{\beta}_{B}$, and $p_{A}^{*}<p^{*}<$ $p_{B}^{*}$. In general allowing for different demand and supply functions in the two original markets implies $\delta=-\frac{\Psi_{A} s_{A}^{\prime}\left(p^{*}\right)+\Psi_{B} s_{B}^{\prime}\left(p^{*}\right)}{D_{A}^{\prime}\left(p^{*}\right)+D_{B}^{\prime}\left(p^{*}\right)}$ which does not trivially compare with $\delta_{A}$ and $\delta_{B}$. However, assuming linear demand functions $D_{A}(\cdot)$ and $D_{B}(\cdot)$ with slopes $D_{A}^{\prime}$ and $D_{B}^{\prime}$ and a common linear $s(\cdot)$ with slope $s^{\prime}$ we have $\delta_{A}=-\frac{\Psi_{A} s^{\prime}}{D_{A}^{\prime}}, \delta_{B}=-\frac{\Psi_{B} s^{\prime}}{D_{B}^{\prime}}$ and

$$
\delta=-\frac{\Psi_{A} s^{\prime}+\Psi_{B} s^{\prime}}{D_{A}^{\prime}+D_{B}^{\prime}}=\frac{1}{\frac{\Psi_{A}}{\Psi} \frac{1}{\delta_{A}}+\frac{\Psi_{B}}{\Psi} \frac{1}{\delta_{B}}} .
$$

Under such assumptions stability (resp. instability) of the integrated market immediately follows from stability (resp. instability) of the original ones:

$$
\bar{\beta} \delta=\left(\frac{\Psi_{A}}{\Psi} \bar{\beta}_{A}+\frac{\Psi_{B}}{\Psi} \bar{\beta}_{B}\right) \frac{1}{\frac{\Psi_{A}}{\Psi} \frac{1}{\delta_{A}}+\frac{\Psi_{B}}{\Psi} \frac{1}{\delta_{B}}} \gtreqless \frac{\frac{\Psi_{A}}{\Psi} \frac{1}{\delta_{A}}+\frac{\Psi_{B}}{\Psi} \frac{1}{\delta_{B}}}{\frac{\Psi_{A}}{\Psi} \frac{1}{\delta_{A}}+\frac{\Psi_{B}}{\Psi} \frac{1}{\delta_{B}}}=1
$$

if $\bar{\beta}_{A} \delta_{A} \gtreqless 1, \bar{\beta}_{B} \delta_{B} \gtreqless 1 .^{7}$ It is important to remark however, that this needs not be the case if less is assumed about the demand and the supply functions. Here is an example

\footnotetext{
${ }^{7}$ Observe that this reflects a well known equality involving arithmetic and harmonic means. Namely, that the harmonic mean of given non-zero numbers $a_{1}, \ldots, a_{n}$ times the arithmetic mean of $a_{1}^{-1}, \ldots, a_{n}^{-1}$ equals 1. See e.g. Hardy et al. [15], p. 14.
} 
in which stability in the original markets is not robust to market integration (see Figure 1):

$$
\begin{array}{|l|l|l|}
\hline S(p)=\tanh (p-1)+1 & D_{A}(p)=\frac{9-5 \ln 2}{10}-\frac{1}{2} p & D_{B}(p)=\frac{21+5 \ln 2}{10}-\frac{1}{2} p \\
\hline p_{A}^{*}=1-\ln 2 & p_{B}^{*}=1+\ln 2 & p^{*}=1 \\
\hline \delta_{A}=\delta_{B}=2 S^{\prime}(1-\ln 2)=\frac{32}{25} & \delta=\frac{2 S^{\prime}(1)}{\frac{1}{2}+\frac{1}{2}}=2 & \bar{\beta}_{A}=\bar{\beta}_{B}=\frac{5}{8} \\
\Rightarrow \delta_{A} \bar{\beta}_{A}=\delta_{B} \bar{\beta}_{B}=\frac{4}{5}<1<\delta \bar{\beta}=\frac{5}{4} .
\end{array}
$$

Insert Figure 1 here

Analogous examples where unstable markets integrate into a stable larger market can also be given. One may wonder whether the result in our example is driven by the change of concavity in the supply. To clarify this point we focus on the case where demand and supply in the two original markets, A and B, differ only by a scale factor. So we have

$$
\begin{array}{ll}
S_{A}(\cdot)=\Psi_{A} s(\cdot), & D_{A}(\cdot)=\Theta_{A} d(\cdot) \\
S_{B}(\cdot)=\Psi_{B} s(\cdot), & D_{B}(\cdot)=\Theta_{B} d(\cdot)
\end{array}
$$

with $s^{\prime}(\cdot)>0$ and $d^{\prime}(\cdot)<0$. As before

$$
\delta_{A}=-\frac{\Psi_{A}}{\Theta_{A}} \frac{s^{\prime}\left(p_{A}^{*}\right)}{d^{\prime}\left(p_{A}^{*}\right)}, \quad \delta_{B}=-\frac{\Psi_{B}}{\Theta_{B}} \frac{s^{\prime}\left(p_{B}^{*}\right)}{d^{\prime}\left(p_{B}^{*}\right)}
$$

Proposition 6. Assume that $\delta_{A} \bar{\beta}_{A}<1, \delta_{B} \bar{\beta}_{B}<1$ and $\frac{\Psi_{A}}{\Theta_{A}}>\frac{\Psi_{B}}{\Theta_{B}}$. If either

$$
s^{\prime}(p) d^{\prime \prime}(p)-s^{\prime \prime}(p) d^{\prime}(p) \geq 0 \quad \forall p \in\left[p_{A}^{*}, p_{B}^{*}\right] \quad \wedge \quad \frac{\Psi_{A} / \Theta_{A}}{\Psi_{B} / \Theta_{B}} \delta_{B} \bar{\beta}_{A}<1
$$

or, alternatively

$$
s^{\prime}(p) d^{\prime \prime}(p)-s^{\prime \prime}(p) d^{\prime}(p) \leq 0 \quad \forall p \in\left[p_{A}^{*}, p_{B}^{*}\right] \quad \wedge \quad \frac{\Psi_{B} / \Theta_{B}}{\Psi_{A} / \Theta_{A}} \delta_{A} \bar{\beta}_{B}<1
$$

then $\delta \bar{\beta}<1$ and hence stability carries through to the integrated market.

The above proposition shows some of the possible extra requirements that guarantee that stability be robust to market integration. An example in which things are easy is when $d(p)=p^{-k}, s(p)=p^{h}, k, h>0$ because in this case $\delta$ is a constant equal to $\frac{h}{k}$.

Observe that the assumption $s^{\prime}(p) d^{\prime \prime}(p)-s^{\prime \prime}(p) d^{\prime}(p) \stackrel{(\leq)}{\geq} 0$, which ensures that the price equilibrium map is monotone, is the same as $d^{\prime \prime}(p) / d^{\prime}(p) \stackrel{(\geq)}{\leq} s^{\prime \prime}(p) / s^{\prime}(p)$ which in turn means that the elasticity of $d^{\prime}(p)$ has to be smaller (larger) than the elasticity of $s^{\prime}(p)$. Further, notice the role played by each market's specific parameters in the technical condition $\frac{\Psi_{A} / \Theta_{A}}{\Psi_{B} / \Theta_{B}} \delta_{B} \bar{\beta}_{A}<1\left(\frac{\Psi_{B} / \Theta_{B}}{\Psi_{A} / \Theta_{A}} \delta_{A} \bar{\beta}_{B}<1\right)$, which imposes a cross-market constraint on the parameters compatible with persistence of stability under aggregation. 


\section{Uncertainty about types and the probability of stability}

In the present paper the number of different types of firms operating in the market is a measure of behavioural heterogeneity. We have already addressed, in Section 3, the issue of understanding what kind of consequences are to be expected on the system's stability if the level of heterogeneity in the market changes as a result of policies or exogenous structural breaks. In the above analysis though, knowledge of the existing types of firms has been given for granted. What happens if uncertainty is introduced about the types of firms acting on the market? Can we still say something about the effects of changing the amount of heterogeneity on stability? It turns out that an observer who is unaware of the level of behavioral heterogeneity in the present context, but knows the structural details of the market (embedded in the $\delta$ parameter) can still make some meaningful predictions regarding the implications of changing the amount of heterogeneity in terms of the likelihood of convergence toward the steady state price.

Consider, for example, a market with a single type of firm. Suppose that the behavioural parameter $\beta$ is unknown and that it can be considered as the realization of a random variable uniformly distributed on the unit interval, $U(0,1)$. Stability is warranted in this case if $\delta \beta<1$ (see (4)), so the probability of such event, for a given structural degree of instability $\delta>1$, will be $\int_{0}^{1 / \delta} d x=\frac{1}{\delta}$. One may wonder how this probability will be affected if $n>1$ or more in general if $n$ varies. We shall define a stable sample of behavioural parameters as one for which the corresponding system (3) has a locally stable steady state $\left(p^{*}\right)$, which means, thanks to the characterisation provided by Proposition 2 , that $\delta \bar{\beta}_{n}<1$. We look for the probability of drawing a stable sample as a function of $\delta$, for a given $n$. Assuming that the $\beta_{i}$ are drawn independently from $U(0,1)$ the expected value of $\bar{\beta}_{n}$ is $1 / 2$. This means the value $\delta=2$ makes the expected value of $\delta \bar{\beta}_{n}$ equal 1. But because the distribution of $\bar{\beta}_{n}$ is symmetric the probability of a stable sample when $\delta=2$ is exactly $1 / 2$. Notice that in this case using the known form of the density for $\bar{\beta}_{n}, f_{n}(x)$, we can write down the explicit probability functions for any $n$, mapping values of $\delta$ with the probability of a stable sample:

$$
P_{n}(\delta)=\int_{0}^{1 / \delta} f_{n}(x) d x
$$

so we can write the probability functions explicitly ${ }^{8}$. These functions are obviously decreasing in $\delta$. Figure 2 depicts such functions for various $n$. Notice that heterogeneity appears to have a stabilising/destabilising impact depending on whether $\delta$ is less/more than a critical value ( 2 in this case). Some sort of polarization effect seems to be at work. Indeed we show that both these observations generalise easily beyond this example based on the uniform distribution. First, because of the stability condition $\delta \bar{\beta}_{n}<1$, the probability of a stable $n$-sample is the probability of having $\bar{\beta}_{n}<1 / \delta$, which decreases with $\delta$ irrespective of the population distribution. Second, we show that polarization is robust, using an argument based on what happens taking the limit for $n \rightarrow \infty$ in a fairly general setting.

\footnotetext{
${ }^{8}$ The density takes the form $\frac{\sum_{k=0}^{n}(-1)^{k}\left(\begin{array}{l}n \\ k\end{array}\right)(x-k)^{n-1} \operatorname{sign}(x-k)}{2(n-1) !} . P_{n}(\delta)$ can be easily worked out if $n$ is not too large. See the working paper version for details.
} 
Proposition 7. Let $f_{n}(x)$ the density for $\bar{\beta}_{n}$ as the result of sampling the $\beta_{i}$ from some distribution over the unit interval with $E\left(\bar{\beta}_{n}\right)=\bar{\beta}$, and $P_{n}(\delta)$ the probability of a stable sample. As $n \rightarrow \infty, P_{n}(\delta)$ converges pointwise to

$$
P_{\infty}(\delta)=\left\{\begin{array}{ccc}
1 & \text { if } & 1<\delta<1 / \bar{\beta} \\
0 & \text { if } & \delta \geq 1 / \bar{\beta}
\end{array}\right.
$$

The above proposition entails that increasing $n$ has the effect of making stability or instability (depending on $\delta$ ) more and more likely. Figure 2 witnesses this fact quite clearly.

\section{Insert Figure 2 here}

Another example, useful to illustrate the polarization effect, is as follows: imagine to draw a sample of $n$ values for the $\alpha_{i}$. The expected value of $\bar{\beta}_{n}$ in this case is $E\left(\sum_{i=1}^{n} \phi_{i} \frac{\alpha_{i}}{2-\alpha_{i}}\right)=\sum_{i=1}^{n} \phi_{i} E\left(\frac{\alpha_{i}}{2-\alpha_{i}}\right)=\int_{0}^{1} \frac{\alpha_{i}}{2-\alpha_{i}} d \alpha_{i}=\ln 4-1 \simeq 0.39$ which shows that sampling these behavioural parameters instead of the instability degrees returns a distribution for $\bar{\beta}_{n}$ more geared towards low values. In principle it would be possible to work out the distribution for $\bar{\beta}_{n}$, just as above: since it does not add much insight (while the algebra is more tedious), we just provide a (numerically obtained) picture similar to the first example, in Figure 3.

\section{Insert Figure 3 here}

\section{Endogenous selection of adaptive parameters}

This section considers an evolutionary mechanism as a device to implement the choice of the predictor by each firm, which therefore ceases to be exogenous to the model. Brock and Hommes [3] and the related branch of literature largely inspire the exercise we conduct in this section.

We have $n$ firms and $k$ predictors consisting each in a different kind of value for the adaptive parameter alpha. In period $t$ each firm evaluates the relative performance of each of the different alphas in terms of forecasting error, given the last observation for the price and the firm's prediction at time $t-1$. Based on these fitness measures the firms calculate probabilities of choosing each predictor by means of the discrete choice model as in Brock and Hommes [3], using a given intensity of choice parameter $\gamma$, measuring how sensitive the firms are to the predictors past performance. Formally, for firm $j$, the probability of choosing predictor $i$ is

$$
\frac{\exp \left(-\gamma\left(p_{j, i}^{e}-p\right)^{2}\right)}{\sum_{i=1}^{k} \exp \left(-\gamma\left(p_{j, i}^{e}-p\right)^{2}\right)}
$$

where $p_{j, i}^{e}=p_{j}^{e}+\alpha_{i}\left(p_{-1}-p_{j}^{e}\right)$ is the price forecast generated by predictor $i$ for firm $j$, using $j$ 's forecast and price of the previous period (we dropped the time index for convenience). The remaining structure of the above model is unchanged.

Observe that adaptive expectations require adding a fraction of one's own forecasting error to one's latest prediction, while here firms are assumed to choose among different 
predictors with probabilities matching the relative precision of the predictors at the antecedent date. Therefore we cannot consider firms types as a whole as in the previous sections, since using the same alphas does not imply generating the same point prediction. As a consequence, we have to keep track of the predictions of each individual firm in order to figure out how the next price will be generated.

As a further consequence remark that in this case one needs to distinguish the probabilities used by each firm in choosing a predictor from the empirical fractions of firms using each predictor. Probabilities and fractions can instead be considered the same in Brock and Hommes [3], where such possibility (arising from the nature of the predictors considered) permits to analyze the model in an entirely deterministic way. On the contrary, the model in the present case remains stochastic. This leads us to consider this extension of the model from a numerical perspective only.

Let demand and supply be linear and the number of predictors sufficiently large to represent fairly well the possible types of adaptive expectations, i.e. from a low alpha that mimics quasi-static expectations to a value large enough to resemble naive (myopic) expectations. We took such number to be equal $10,{ }^{9}$ so that the alphas among which to choose were $.05, .15, \ldots, .85, .95$. There are various effects in the model that are worth studying, in particular the effect of the intensity of choice, given a fixed value of $\delta$ as in Brock and Hommes [3], as well as the effect of increasing $\delta$ given a fixed value for the intensity of choice. Then there is also the effect of the number of firms given the other parameters. The results are summarized in Figures 4 to 8. A variety of dynamic behaviors as a function of those parameters can be observed ${ }^{10}$.

First consider, as we did in Section 4, what happens when $\gamma$ is fixed and $\delta$ and $n$ vary (we focused on the cases of $n$ equal 2,3,5,10, and 100 as in Section 4, whereas it is assumed $\gamma=3$; see Figure 4).

\section{Insert Figure 4 here}

Low values of $\delta$ are unsurprisingly associated with frequent converging behavior and this feature gets stronger when $n$ is increased. Intermediate values for $\delta$ entail a variety of different possibilities. For small values of $n$ an interesting type of behavior emerges rather frequently, in which the price alternates a quasi-convergent regime to periods of much higher volatility; in this case the price time series displays booms and busts. When $n$ is higher we observe only few of these cases, but instead we have quasi-periodic time series with an approximately constant volatility. As $\delta$ is further increased we massively observe divergence for low $n$ and quasi-periodicity for large $n$. Some examples are reported in Figure 8.

\section{Insert Figure 8 here}

It is remarkable that in this richer context the number of firms retains a decisive role in shaping the dynamics of the system. In particular, low and moderate values for

\footnotetext{
${ }^{9}$ Increasing this value did not impact significantly on the results. Being significantly more parimonious (e.g. two or three different alphas) instead imposed us to make arbitrary choices about the actual values chosen for the different alphas and results were overly sensitive to such arbitrary choices.

${ }^{10}$ The simulations were carried out considering a time span of length $T=300$. The simulations entail a random initial choice for price and expectations (chosen to be in the vicinity of the steady state), and they were replicated 20000 times for each combination of the $m, \delta$ and $\omega$ parameters.
} 
$n$ are compatible with rich dynamics: convergence, explosive paths, booms and busts, quasi-periodic motions are all possibilities. Such richness was not possible in our original setup with fixed predictors under linearity, whereby obviously there could only be either convergence to or divergence from the unique fixed point. As $n$ increases, a form of polarization emerges in this context too, but remarkably and as a difference with respect to the static case, divergence is not a possible asymptotic outcome. Instead for high values of $\delta$ and $n$, quasi-periodic dynamics becomes prevalent. So, the evolutionary setup induces some kind of stabilization on the system, in the sense that the price oscillations get to be bounded when the amount of heterogeneity is sufficiently high.

Turning to a different exercise, namely that of fixing a value for $\delta$ and letting the intensity of choice to vary, we have a way of checking the robustness of the finding from Proposition 4 of Section 2.3. Indeed we repeated this exercise for three different values of $\delta$ :

- $\delta=2$ for which the model with no switching would be stable;

- $\delta=2.7$ for which the model with no switching would be unstable but it would be stable if we erase the heterogeneity from the model considering a representative firm having a value of alpha equal to the mean of the alphas ( $1 / 2$ in this case);

- $\delta=3.5$ for which, with no switching, both the heterogeneous model and its representative firm counterpart would be unstable.

\section{Insert Figures 5, 6 and 7 here}

The results are as follows. When $\delta=2$ there are only two possibilities: either we observe convergence to or divergence from (explosive paths) the fixed point. Divergence is more likely for low values of $n$ and for larger values of the intensity of choice, $\gamma$. So in this case, allowing for endogenous switching does not alter much the long run outcomes with respect to those of the model with fixed predictors, provided the intensity of choice is not too large or there is sufficient heterogeneity. A similar situation arises when $\delta=2.7$ (this value has been used in Brock and Hommes [3] as well), save the fact that the balance between divergent and convergence behavior bends more towards divergence with respect to the case with lower $\delta$. When $\delta=3.5$ we see the emergency of other types of dynamical behavior beside convergence and divergence.

Finally, a general conclusion emerging from the simulations is that, when the number of firms increases and independently on the value of $\delta$, the divergent behavior tends to disappear. Conditional on the values of $\gamma$ and $\delta$, convergent or quasi-periodic behavior will dominate asymptotic dynamics. Hence it appears that the endogenous switching mechanism tends to transform the polarization between convergence and divergence observed in the previous section into a polarization between convergence and quasi-periodic price oscillations.

\section{Conclusions}

We have analysed the dynamic consequences of expectations heterogeneity in a fairly general cobweb model with $n$ types of firms, each resorting to adaptive expectations 
with a specific gain parameter. The concepts of structural and behavioural degree of instability were introduced to distinguish the different possible sources of failures to converge to the unique steady state in the model. In particular the behavioural degree of instability depends exclusively on the sensitivity of firms' expectations. Stability is shown to obtain if and only if the product of the two sources of instability is less than one. Within the model, we have clarified how marketwise outcomes are grounded in individual firms' characteristics and how a representative agent assumption can inaccurately predict a stable outcome when the whole heterogeneous picture implies otherwise. This is one aspect in which expectations heterogeneity is shown to matter.

The issue of how the stability conditions modify if the number of types vary has also been investigated in a comparative statics analysis. Further, conditions that make stability robust to market aggregation, where such changes in the amount of heterogeneity are bound to arise in practice, are also provided in the paper.

The basic model has then been extended in order to assume the point of view of an authority who is uncertain about the firms types. Positing a probability distribution over possible types active in the market, the structural degree of instability is shown to determine how heterogeneity affects the probability of ending up with a stable outcome, for given number of firms types and structural degree of instability. A form of polarization is documented, by which when the number of types is large, stability most likely obtains for levels of the structural degree of instability up to a certain threshold, while instability is almost certain past the threshold. The amount of the heterogeneity and its possible variations play a critical role in shaping the range of possible long-run outcomes of the model.

A further extension we pursued was aimed at one obvious limitation of the model, namely the fact that the firms do not update their expectation mechanism. The choice of predictors was therefore made endogenous through a discrete choice based evolutionary mechanism, along the lines of Brock and Hommes [3]. Heterogeneity matters and is persistent in this context: conditions implying divergent dynamics in the baseline model produce different outcomes in the evolutionary setup. For low values of the structural degree of instability a rational route to market stability arises in a world with only adaptive expectations and all rules available for free, while for higher values of the structural degree of instability the dynamics may show excess volatility (especially when agents do not stick to their behavioural rule). Moreover increasing the behavioural heterogeneity undercuts the possible asymptotic behaviours one is bound to observe: in particular for parametric configurations that lead to (or are compatible with) explosive price paths, increasing the amount of heterogeneity rules out such divergent behaviour leaving only the possibility of convergence toward the steady state or quasi-periodic oscillations around it.

The analysis carried out in this article leads to several additional research questions, regarding the robustness of our results to changes in various institutional and parametric assumptions. In particular the role of a subset of firms having rational expectations or using other expectation mechanisms beside the adaptive ones might be investigated. A further direction of research suggests modelling explicitly the possibility of market entry and exit of producers: this, while technically challenging, as it requires being more specific on institutional features such as borrowing contraints and on the nature of the firms and the productive process, would considerably enrich the model and its realism. 


\section{Acknowledgements}

We wish to thank Marcello Galeotti for a very useful suggestion in the early phase of this work. We also thank Cars Hommes and three anonymous referees for helpful comments.

\section{Appendix}

\section{Proof of Lemma 1.}

i) Consider first the simpler case in which $c_{1}=\cdots=c_{n}=1$. Observe that

$$
\begin{aligned}
\operatorname{det} M & =\operatorname{det} N \\
N & =\left(\begin{array}{cccc}
a_{1}+b_{1} & -b_{1} & \cdots & -b_{1} \\
a_{2} & b_{2} & \cdots & 0 \\
\vdots & \vdots & \ddots & \vdots \\
a_{n} & 0 & \cdots & b_{n}
\end{array}\right)
\end{aligned}
$$

where $N$ is obtained from $M$ subtracting its first column from the remaining columns. The equality of the two determinants stems from multilinearity in columns. Developing the determinant of $N$ along the first column clearly shows that it is linear in each $a_{i}$. As a consequence, expressing $\operatorname{det} M$ as sum of products along permutations of the column indices, the only terms that do not cancel out are contained in the product of terms along the diagonal (any other permutation contains products of the type $a_{i} a_{j}, i \neq j$. Eliminating terms that involve such products between different $a_{i}$ 's from the product along the diagonal gives the required result. Now let

$$
A=\left(\begin{array}{cccc}
a_{1} & a_{1} & \cdots & a_{1} \\
a_{2} & a_{2} & \cdots & a_{2} \\
\vdots & \vdots & \ddots & \vdots \\
a_{n} & a_{n} & \cdots & a_{n}
\end{array}\right), B=\left(\begin{array}{cccc}
b_{1} & 0 & \cdots & 0 \\
0 & b_{2} & \cdots & 0 \\
\vdots & \vdots & \ddots & \vdots \\
0 & 0 & \cdots & b_{n}
\end{array}\right), C=\left(\begin{array}{cccc}
c_{1} & 0 & \cdots & 0 \\
0 & c_{2} & \cdots & 0 \\
\vdots & \vdots & \ddots & \vdots \\
0 & 0 & \cdots & c_{n}
\end{array}\right)
$$

For values of the $c_{i} \neq 1$ the same results applies remarking that

$$
M=A C+B=\left(A+B C^{-1}\right) C
$$

therefore $\operatorname{det} M=\left(\prod_{i=1}^{n} \frac{b_{i}}{c_{i}}+\sum_{i=1}^{n} a_{i} \prod_{j \neq i} \frac{b_{j}}{c j}\right) \prod_{i=1}^{n} c_{i}=\prod_{i=1}^{n} b_{i}+\sum_{i=1}^{n} c_{i} a_{i} \prod_{j \neq i} b_{j}$ as stated.

ii) Using $i)$ the characteristic polynomial of $M$ writes as

$$
P(\lambda)=\prod_{i=1}^{n}\left(b_{i}-\lambda\right)+\sum_{i=1}^{n} c_{i} a_{i} \prod_{j \neq i}\left(b_{j}-\lambda\right)
$$

Suppose that $b_{1}>b_{2}>\cdots>b_{n}$. Then

$$
\begin{aligned}
n \text { even } & \Rightarrow P\left(b_{1}\right)>0, P\left(b_{2}\right)<0, \ldots, P\left(b_{n}\right)<0 \\
n \text { odd } & \Rightarrow P\left(b_{1}\right)<0, P\left(b_{2}\right)>0, \ldots, P\left(b_{n}\right)<0
\end{aligned}
$$


Therefore $P(\lambda)$ has $n-1$ real roots and hence $n$ real roots. Suppose more in general that the set of $b_{i} \mathrm{~s}$ is as follows:

$$
\{\underbrace{b_{1}, \ldots, b_{1}}_{n_{1} \text { times }}, \underbrace{b_{2}, \ldots, b_{2}}_{n_{2} \text { times }}, \ldots, \underbrace{b_{m}, \ldots, b_{m}}_{n_{m} \text { times }}\}
$$

with $\sum_{j=1}^{m} n_{j}=n$. Let also $k_{1}=\left\{1, \ldots, n_{1}\right\}, k_{2}=\left\{n_{1}+1, \ldots, n_{1}+n_{2}\right\}, \ldots, k_{m}=$ $\left\{\sum_{j=1}^{m-1} n_{j}+1, \ldots, n\right\}$ and $\bar{a}_{i}=\sum_{j \in k_{i}} c_{j} a_{j}$. Then

$$
\begin{aligned}
P(\lambda) & =\prod_{i=1}^{m}\left(b_{i}-\lambda\right)^{n_{i}}+\sum_{i=1}^{n} \bar{a}_{i}\left(b_{i}-\lambda\right)^{n_{i}-1} \prod_{j \neq i}\left(b_{j}-\lambda\right)^{n_{j}} \\
& =\prod_{i=1}^{m}\left(b_{i}-\lambda\right)^{n_{i}-1}\left(\prod_{i=1}^{m} b_{i}+\sum_{i=1}^{n} \bar{a}_{i} \prod_{j \neq i}\left(b_{j}-\lambda\right)\right) \\
& \equiv P_{1}(\lambda) P_{2}(\lambda)
\end{aligned}
$$

where, counting multiplicity, $P_{1}(\lambda)$ has $n-m$ real roots and $P_{2}(\lambda)$ has $m$ real roots (this stems from what we showed for the case of distinct $b_{i} \mathrm{~s}$ ).

iii) and $i v)$ From (11) we have that $n-m$ eigenvalues take values in $\left\{b_{i}\right\}_{i=1, \ldots, n}$. Also, from (10), it follows that $m-1$ eigenvalues belong to $\left(b_{n}, b_{1}\right)$. Finally, as $P\left(b_{n}\right)<0$ and $\lim _{\lambda \rightarrow-\infty} P(\lambda)=+\infty$, the remaining root of $P(\lambda)$ must be smaller than $b_{n}$ and therefore it is greater than -1 if and only if $P(-1)>0$.

Proof of Proposition 2. Recalling that Jacobian (5) is a particular specification of matrix (6) with $a_{i}=-\alpha_{i}, b_{i}=1-\alpha_{i}, c_{i}=\phi_{i} \delta$, Lemma 1 part $i v$ ) states that local stability is equivalent to having the characteristic polynomial positive when evaluated at $-1, P(-1)>0$. In the case of matrix $J_{n}$ we have

$$
\begin{aligned}
P_{n}(-1) & =\prod_{j=1}^{n}\left(2-\alpha_{j}\right)-\delta \sum_{i=1}^{n} \alpha_{i} \phi_{i} \prod_{j \neq i}\left(2-\alpha_{j}\right) \\
& =\prod_{j=1}^{n}\left(2-\alpha_{j}\right)\left(1-\delta \sum_{i=1}^{n} \phi_{i} \frac{\alpha_{i}}{2-\alpha_{i}}\right) \\
& =\prod_{j=1}^{n}\left(2-\alpha_{j}\right)\left(1-\delta \bar{\beta}_{n}\right)
\end{aligned}
$$

which is positive if and only if $\delta \bar{\beta}_{n}<1$.

Proof of Proposition 3. Following the same argument in the proof of part iv) of Lemma $1, \lambda_{\min }$ is greater than 0 if and only if the characteristic polynomial, $P(\lambda)$, is positive at $\lambda=0$. As

$$
P(0)=\prod_{i=1}^{n}\left(1-\alpha_{i}\right)-\sum_{i=1}^{n} \alpha_{i} \phi_{i} \delta \prod_{j \neq i}\left(1-\alpha_{j}\right)=\prod_{i=1}^{n}\left(1-\alpha_{i}\right)\left(1-\sum_{i=1}^{n} \frac{\alpha_{i} \phi_{i} \delta}{\left(1-\alpha_{i}\right)}\right)
$$


we have $P(0)>0$ if and only if

$$
\sum_{i=1}^{n} \phi_{i} \frac{\alpha_{i}}{\left(1-\alpha_{i}\right)}<\frac{1}{\delta}
$$

Proof of Proposition 4. Stability conditions for the two cases (see Proposition 2) imply:

$$
\begin{aligned}
& \sum_{i=1}^{n} \phi_{i} \frac{\alpha_{i}}{2-\alpha_{i}}<\frac{1}{\delta} \text { for the heterogeneous market } \\
& \frac{\sum_{i=1}^{n} \phi_{i} \alpha_{i}}{2-\sum_{i=1}^{n} \phi_{i} \alpha_{i}}<\frac{1}{\delta} \text { for the homogeneous market }
\end{aligned}
$$

Observe that the function $f(x)=\frac{x}{2-x}$ is strictly convex in $[0,1]$ so necessarily

$$
\frac{\sum_{i=1}^{n} \phi_{i} \alpha_{i}}{2-\sum_{i=1}^{n} \phi_{i} \alpha_{i}}<\sum_{i=1}^{n} \phi_{i} \frac{\alpha_{i}}{2-\alpha_{i}}
$$

which gives the desired result.

Proof of Lemma 5. Consider the derivative with respect to $\alpha_{i}$ of the characteristic polynomial,

$$
\begin{aligned}
\frac{\partial P(\lambda)}{\partial \alpha_{i}} & =\frac{\partial}{\partial \alpha_{i}}\left(\prod_{j=1}^{n}\left(1-\alpha_{j}-\lambda\right)-\sum_{k=1}^{n} \alpha_{k} \phi_{k} \delta \prod_{j \neq k}\left(1-\alpha_{j}-\lambda\right)\right) \\
& =-\prod_{j \neq i}\left(1-\alpha_{j}-\lambda\right)-\phi_{i} \delta \prod_{j \neq i}\left(1-\alpha_{j}-\lambda\right)+\sum_{k \neq i} \alpha_{k} \phi_{k} \delta \prod_{j \neq k, i}\left(1-\alpha_{j}-\lambda\right)
\end{aligned}
$$

Recall that, given Lemma 1 iii), for all $i, \lambda_{\min }<1-\alpha_{i}$ while $\lambda_{\max } \leq 1-\alpha_{\min }$. Whenever $\lambda \neq 1-\alpha_{i}$

$$
\begin{aligned}
\frac{\partial P(\lambda)}{\partial \alpha_{i}} & =\frac{\prod_{j=1}^{n}\left(1-\alpha_{j}-\lambda\right)}{\left(1-\alpha_{i}-\lambda\right)}-\phi_{i} \delta \prod_{j \neq i}\left(1-\alpha_{j}-\lambda\right)+\frac{\sum_{k \neq i} \alpha_{k} \phi_{k} \delta \prod_{j \neq k}\left(1-\alpha_{j}-\lambda\right)}{\left(1-\alpha_{i}-\lambda\right)} \\
& =-\frac{\prod_{j=1}^{n}\left(1-\alpha_{j}-\lambda\right)-\sum_{k=1}^{n} \alpha_{k} \phi_{k} \delta \prod_{j \neq k}\left(1-\alpha_{j}-\lambda\right)}{\left(1-\alpha_{i}-\lambda\right)}-\left(1+\frac{\alpha_{i}}{1-\alpha_{i}-\lambda}\right) \phi_{i} \delta \prod_{j \neq i}\left(1-\alpha_{j}-\lambda\right) \\
& =-\frac{P(\lambda)}{\left(1-\alpha_{i}-\lambda\right)}-\left(1+\frac{\alpha_{i}}{1-\alpha_{i}-\lambda}\right) \phi_{i} \delta \prod_{j \neq i}\left(1-\alpha_{j}-\lambda\right)
\end{aligned}
$$

So evaluating the derivative at $\lambda_{\min }$, we have

$$
\left.\frac{\partial P(\lambda)}{\partial \alpha_{i}}\right|_{\lambda=\lambda_{\min }}=-\left(1+\frac{\alpha_{i}}{1-\alpha_{i}-\lambda_{\min }}\right) \phi_{i} \delta \prod_{j \neq i}\left(1-\alpha_{j}-\lambda_{\min }\right)<0
$$


as $P\left(\lambda_{\min }\right)=0$ and, due to part iii) of Lemma $1,\left(1-\alpha_{j}-\lambda_{\min }\right)>0$ for all $j$. Finally, because $\lim _{\lambda \rightarrow-\infty} P(\lambda)=+\infty$ the result follows from the intermediate value theorem. Besides, at $\lambda_{\max }$, we have

$$
\left.\frac{\partial P(\lambda)}{\partial \alpha_{i}}\right|_{\lambda=\lambda_{\max }}=-\left(1+\frac{\alpha_{i}}{1-\alpha_{i}-\lambda_{\max }}\right) \phi_{i} \delta \prod_{j \neq i}\left(1-\alpha_{j}-\lambda_{\max }\right) \begin{array}{lll}
>0 & \text { if } & n \text { is even } \\
<0 & \text { if } & n \text { is odd }
\end{array}
$$

due to Lemma 1 iii) and $\lambda_{\max } \neq 1-\alpha_{i}$. Again, because $\lim _{\lambda \rightarrow+\infty} P(\lambda)=(-1)^{n} \infty$ the result follows from the intermediate value theorem. It remains to consider the case $\lambda_{\max }=1-\alpha_{\min }$. If $\alpha_{i} \neq \alpha_{\min }$ then trivially

$$
\left.\frac{\partial P(\lambda)}{\partial \alpha_{i}}\right|_{\lambda=\lambda_{\max }}=0
$$

Otherwise if $\alpha_{i}=\alpha_{\min }$ the result directly follows from part iii) of Lemma 1 .

Proof of Proposition 6. Notice: $\frac{\Psi_{A}}{\Theta_{A}}>\frac{\Psi_{B}}{\Theta_{B}} \Rightarrow \frac{\Psi_{B}}{\Theta_{B}}<\frac{\Psi_{A}+\Psi_{B}}{\Theta_{A}+\Theta_{B}}<\frac{\Psi_{A}}{\Theta_{A}}$; also $p_{A}^{*}<p^{*}<p_{B}^{*}$. Assumptions in (7) imply that

$$
\left(-\frac{s^{\prime}(p)}{d^{\prime}(p)}\right)^{\prime}=\frac{-s^{\prime \prime}(p) d^{\prime}(p)+s^{\prime}(p) d^{\prime \prime}(p)}{\left(d^{\prime}(p)\right)^{2}} \geq 0
$$

As a result

$$
\begin{aligned}
\delta \bar{\beta} & =-\frac{\left(\frac{\Psi_{A}+\Psi_{B}}{\Theta_{A}+\Theta_{B}}\right) s^{\prime}\left(p^{*}\right)}{d^{\prime}\left(p^{*}\right)}\left(\frac{\Psi_{A}}{\Psi} \bar{\beta}_{A}+\frac{\Psi_{B}}{\Psi} \bar{\beta}_{B}\right) \\
& \leq\left(\frac{\Psi_{A}+\Psi_{B}}{\Theta_{A}+\Theta_{B}}\right)\left(-\frac{s^{\prime}\left(p_{B}^{*}\right)}{d^{\prime}\left(p_{B}^{*}\right)}\right)\left(\frac{\Psi_{A}}{\Psi} \bar{\beta}_{A}+\frac{\Psi_{B}}{\Psi} \bar{\beta}_{B}\right) \\
& =\left(\frac{\Psi_{A}}{\Theta_{A}+\Theta_{B}} \bar{\beta}_{A}\right)\left(-\frac{s^{\prime}\left(p_{B}^{*}\right)}{d^{\prime}\left(p_{B}^{*}\right)}\right)+\left(\frac{\Psi_{B}}{\Theta_{A}+\Theta_{B}} \bar{\beta}_{B}\right)\left(-\frac{s^{\prime}\left(p_{B}^{*}\right)}{d^{\prime}\left(p_{B}^{*}\right)}\right) \\
& =\frac{\Theta_{A}}{\Theta_{A}+\Theta_{B}}\left(\frac{\Psi_{A}}{\Theta_{A}} \bar{\beta}_{A}\right)\left(-\frac{s^{\prime}\left(p_{B}^{*}\right)}{d^{\prime}\left(p_{B}^{*}\right)}\right)+\delta_{B} \bar{\beta}_{B} \frac{\Theta_{B}}{\Theta_{A}+\Theta_{B}} \\
& <\frac{\Theta_{A}}{\Theta_{A}+\Theta_{B}}+\frac{\Theta_{B}}{\Theta_{A}+\Theta_{B}}=1
\end{aligned}
$$

The proof under the alternative assumptions in (8) is identical.

Proof of Proposition 7. The strong law of large numbers shows that

$$
\operatorname{Pr}\left(\lim _{n \rightarrow \infty} \bar{\beta}_{n}=\bar{\beta}\right)=1 \Rightarrow \operatorname{Pr}\left(\lim _{n \rightarrow \infty} \delta \bar{\beta}_{n}=\delta \bar{\beta}\right)=1
$$

and therefore, for any $\delta>1$,

$$
\operatorname{Pr}\left(\lim _{n \rightarrow \infty} \delta \bar{\beta}_{n}<1\right)=\left\{\begin{array}{ccc}
1 & \text { if } & 1<\delta<1 / \bar{\beta} \\
0 & \text { if } \quad \delta \geq 1 / \bar{\beta}
\end{array}\right.
$$




\section{References}

[1] Branch, W. (2002). Local convergence properties of a cobweb model with rationally heterogeneous expectations. Journal of Economic Dynamics and Control 27, 63-85.

[2] Branch, W. (2004). The Theory of Rationally Heterogeneous Expectations: Evidence from Survey Data on Inflation Expectations. The Economic Journal, 114, 592-621.

[3] Brock, W. \& Hommes, C. (1997). A Rational Route to Randomness. Econometrica, 65, 1059-1095.

[4] Buraschi, A. \& Jiltsov, A. (2007). Model Uncertainty and Option Markets with Heterogeneous Beliefs. Journal of Finance, 61(6), 2841-2897.

[5] Carlson, J.A. (1968). An Invariably Stable Cobweb Model. Review of Economic Studies 35(3), 360-362

[6] Carroll, C.D. (2003). Macroeconomic Expectations of Households and Professional Forecasters. Quarterly Journal of Economics, 118(1), 269-298.

[7] Chavas, J.P. (2000). On information and market dynamics: the case of the U.S. beef market. Journal of Economic Dynamics \& Control, 24, 833-853.

[8] Chiarella, C., He, X.-Z., Hung, H. \& Zhu P. (2006). An analysis of the cobweb model with boundedly rational heterogeneous producers. Journal of Economic Behavior \& Organization 61(4), 750-768.

[9] Colucci, D. \& Valori, V. (2008). Asset price dynamics when behavioural heterogeneity varies. Computational Economics, 32, 3-20.

[10] Commendatore, P. \& Currie M. (2008). The cobweb, borrowing and financial crises. Journal of Economic Behavior \& Organization, 66, 625-640.

[11] Evans, G. W. \& Guesnerie, R. (2005). Coordination on saddle-path solutions: the eductive viewpoint - linear multivariate models. Journal of Economic Theory, 124, 202-229.

[12] Franke, R. \& Nesemann, T. (1999). Two destabilizing strategies may be jointly stabilizing. Journal of Economics, 69(1), 1-18

[13] Frankel, J.A. \& Froot, K.A. (1990). The Rationality of the Foreign Exchange Rate. Chartists, Fundamentalists and Trading in the Foreign Exchange Market, American Economic Review 80(2), AEA Papers and Proceedings, 181-185.

[14] Goeree, J. K. \& Hommes, C. (2000). Heterogeneous beliefs and the non-linear cobweb model, Journal of Economic Dynamics and Control, 24, 761-798. s

[15] Hardy, G., Littlewood, J. E. \& Pòlya, G. (1988). Inequalities. Second Edition. Cambridge University Press.

[16] Honkapohja, S. \& Mitra, K. (2005). Performance of monetary policy with internal central bank forecasting. Journal of Economic Dynamics and Control, 29, 627-658.

[17] Hommes, C. (1994). Dynamics of the cobweb model with adaptive expectations and nonlinear supply and demand. Journal of Economic Behavior \& Organization, 24(3), 315-335.

[18] Hommes, C. (2006). Heterogeneous agent models in economic and finance. Handbook of computational Economics, Volume 2: Agent-based computational economics, Edited by Judd, K.L. \& Tesfatsion, T., Elsevier Science B.V.

[19] Jouini, E. \& Napp, C. (2007). Consensus Consumer and Intertemporal Asset Pricing with Heterogeneous Beliefs. Review of Economic Studies, 74(4), 1149-1174.

[20] Lasselle, L., Svizzero, S. \& Tisdell, C. (2005). Stability And Cycles In A Cobweb Model With Heterogeneous Expectations. Macroeconomic Dynamics, 9, 630-650.

[21] Mankiw, N., Reis, R. \& Wolfers, J. (2004). Disagreement about Inflation Expectations. NBER Macroeconomics Annual 2003, Edited by Gertler, M. \& Rogoff, K, MIT Press.

[22] Manzan, S. \& Westerhoff, F.H. (2007). Heterogeneous expectations, exchange rate dynamics and predictability. Journal of Economic Behavior \& Organization, 64(1), 111-128.

[23] Negroni, G. (2003). Adaptive expectations coordination in an economy with heterogeneous agents. Journal of Economic Dynamics \& Control, 28, 117-140.

[24] Nerlove M. (1958). Adaptive expectations and cobweb phenomena. Quarterly Journal of Economics, $72(2), 227-240$

[25] Onozaki, T., Sieg, G. \& Yokoo, M. (2003). Stability, chaos and multiple attractors: a single agent makes a difference. Journal of Economic Dynamics and Control 27, 1917-1938. 


\section{Figure legends}

Figure 1: Stability for markets considered separately is lost under aggregation.

Figure 2: Probability of a stable sample of betas.

Figure 3: Probability of a stable sample of alphas. 
Figure 4: $\delta$ on $\mathrm{x}$-axis, $\gamma=3$. Top-left: convergence. Top-right: divergence. Bottom: other behaviors.

Figure 5: $\gamma$ on x-axis, $\delta=2$. Left: convergence. Right: divergence. No other behaviors observed.

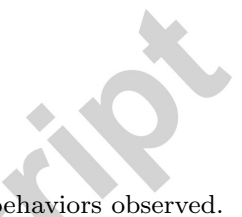

Figure 5: $\gamma$ on x-axis, $\delta=2$. Left: convergence. Right: divergence. No other behaviors observed.

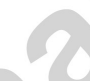

Figure 6: $\gamma$ on x-axis, $\delta=2.7$. Left: convergence. Right: divergence. No other behaviors observed.

Figure 7: $\gamma$ on x-axis, $\delta=3.5$. Left: convergence. Right: divergence. Bottom: other behaviors.

Figure 8: Top down: converging, diverging, quasi-periodic, booms and busts. 
Figures

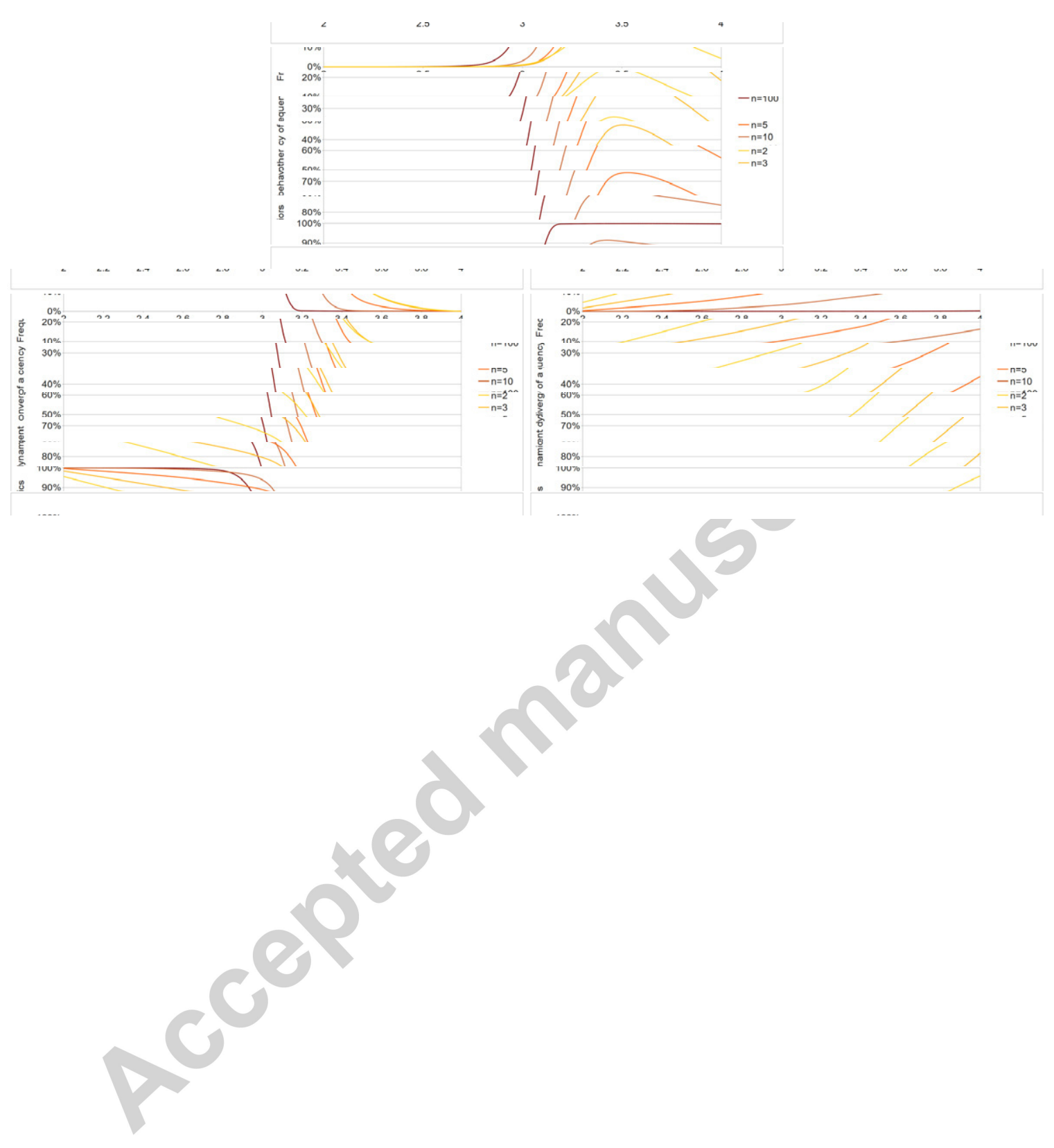



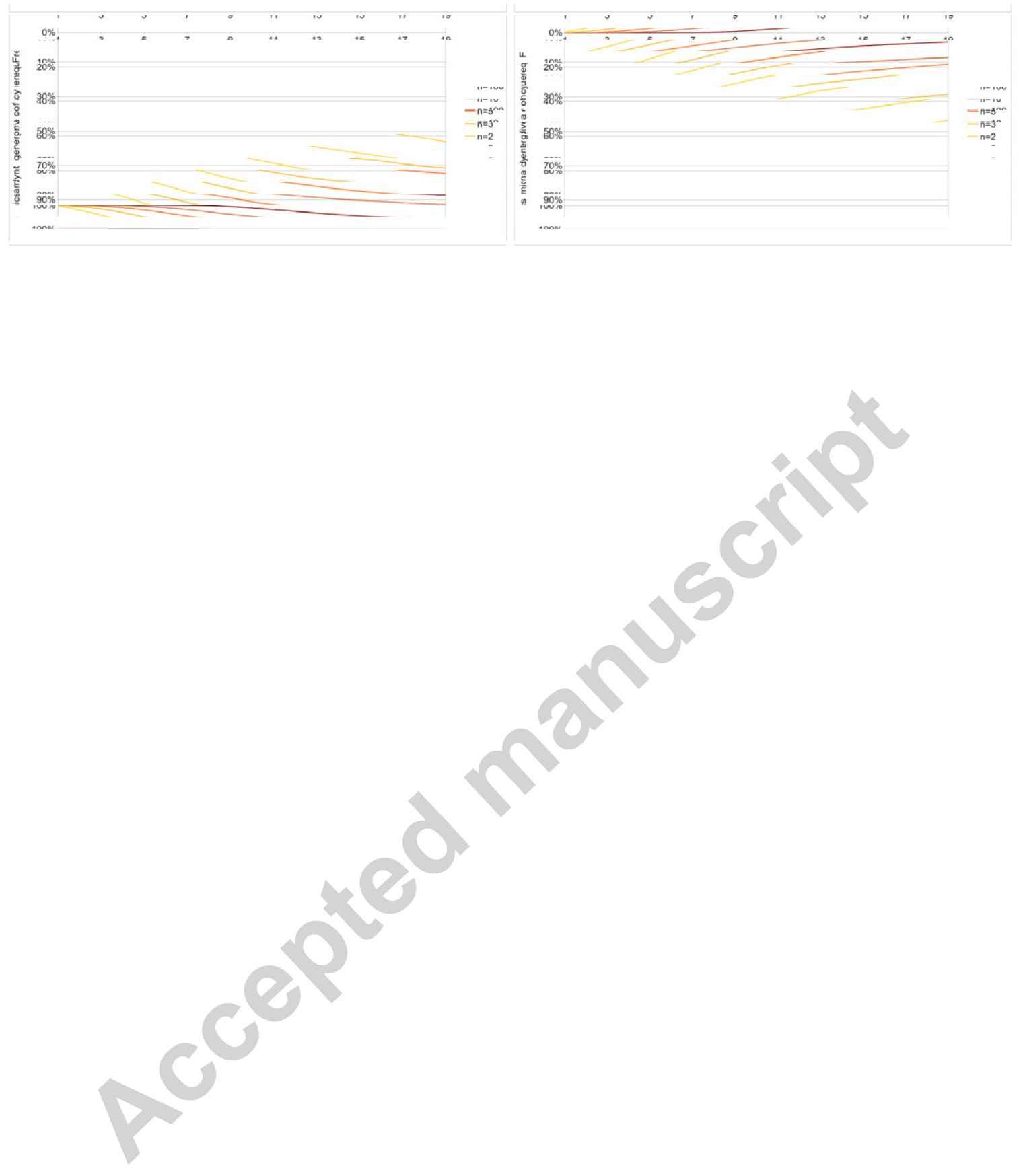

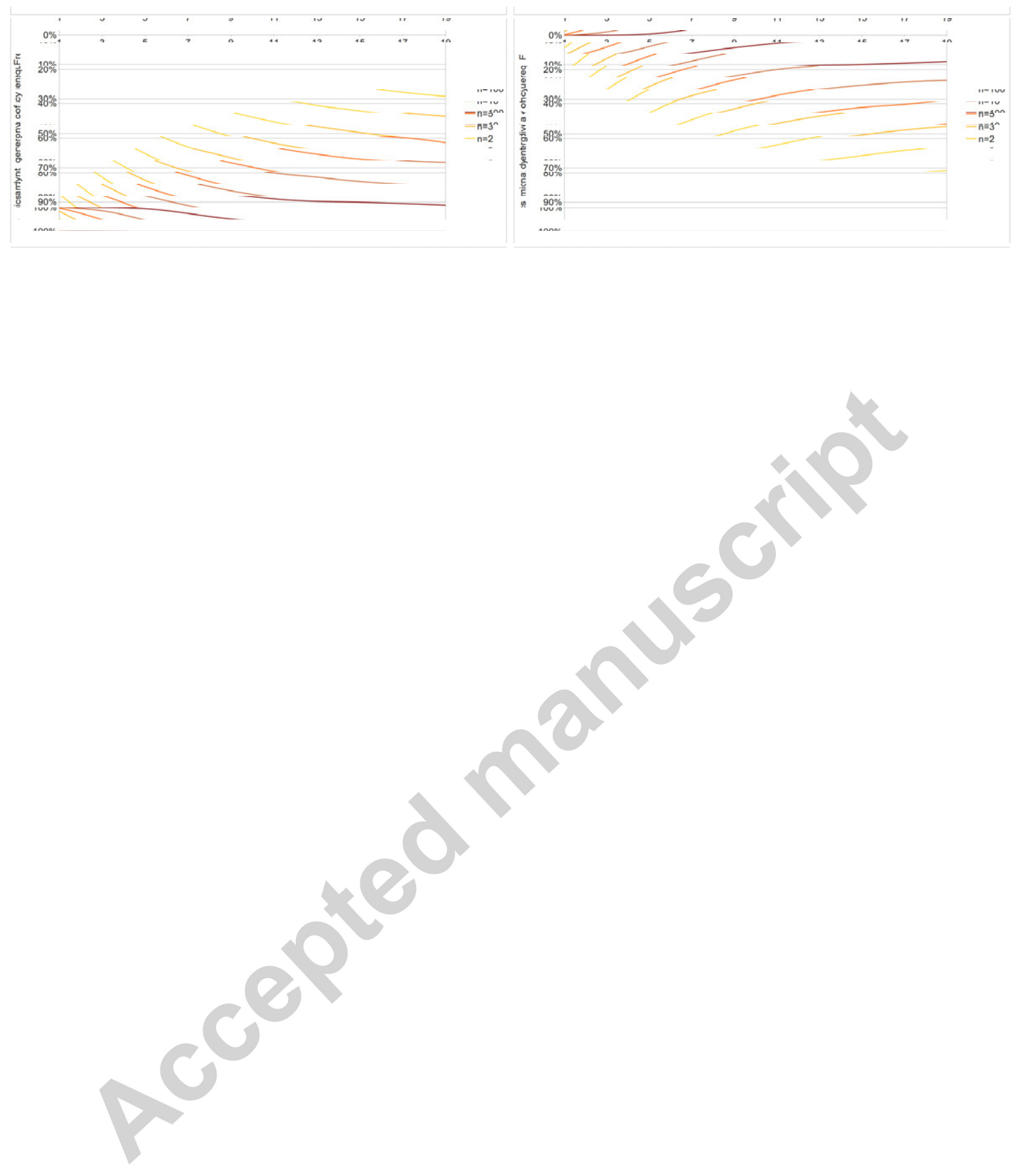


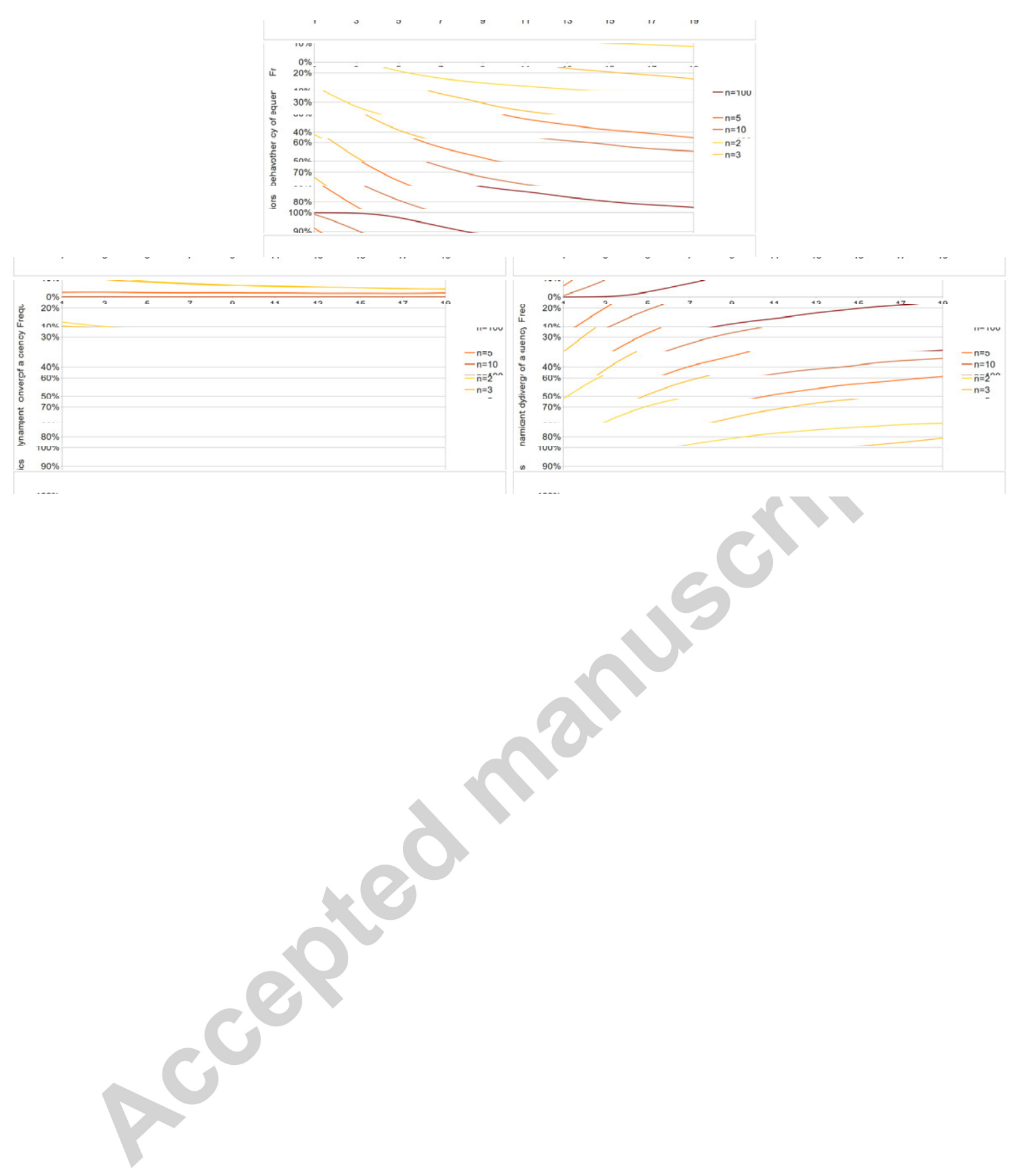



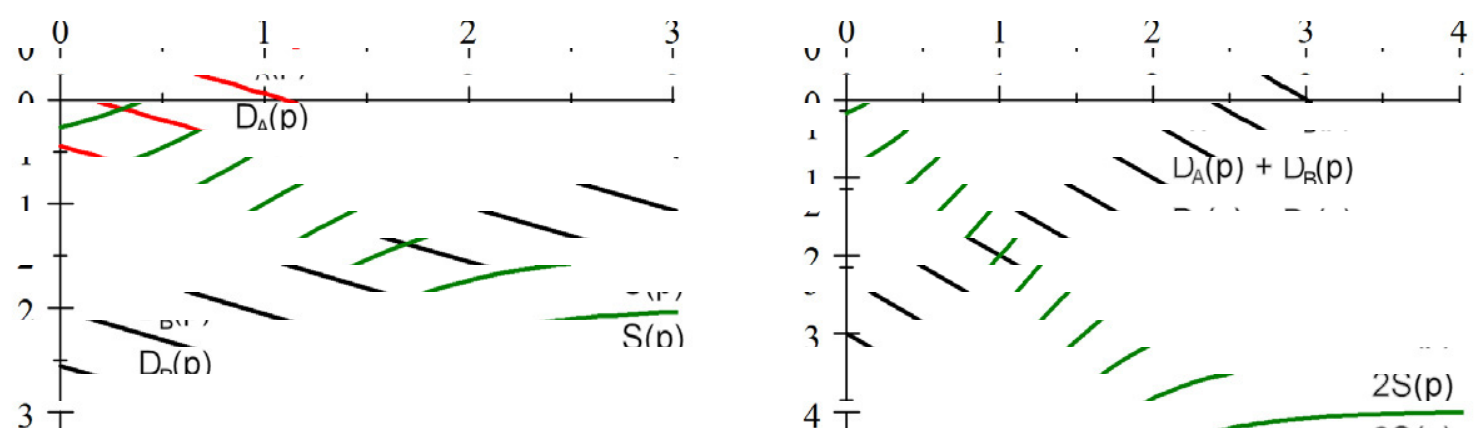


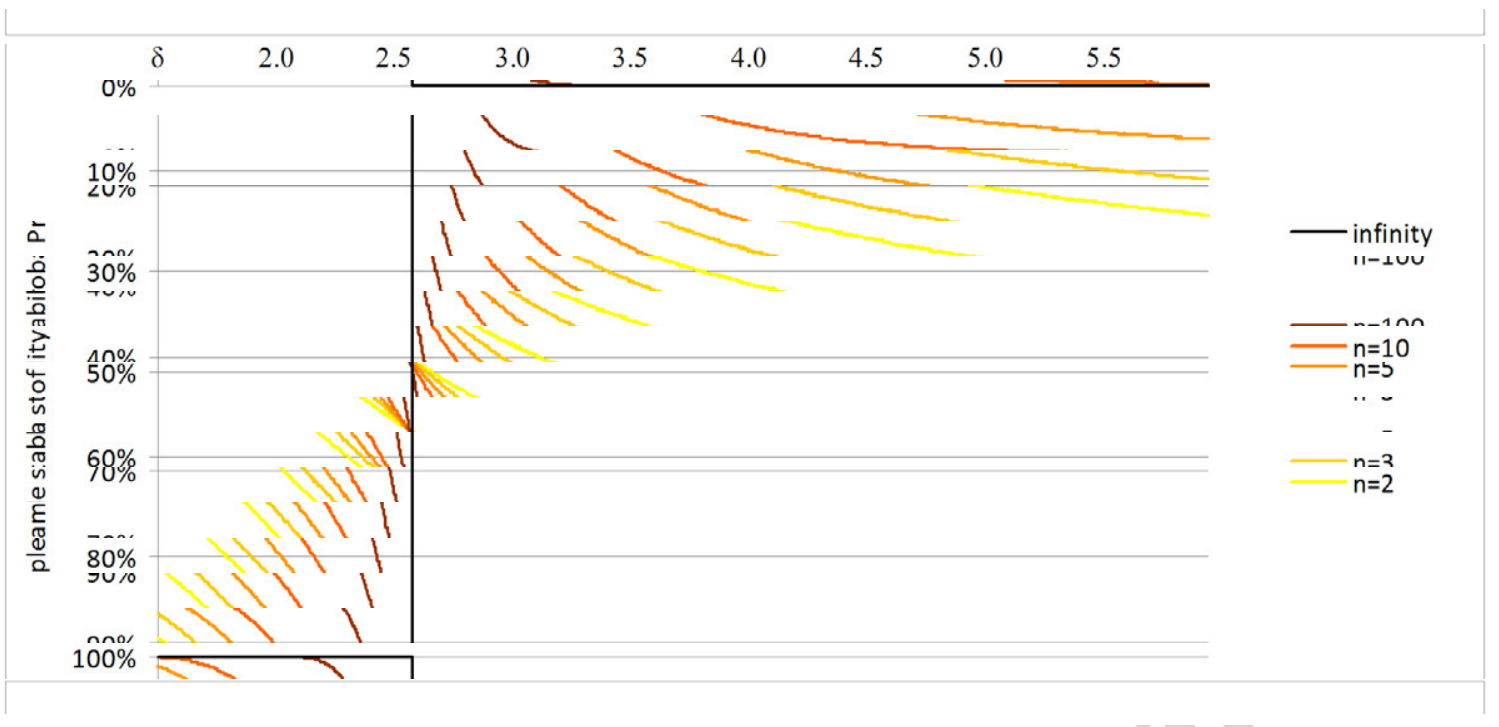




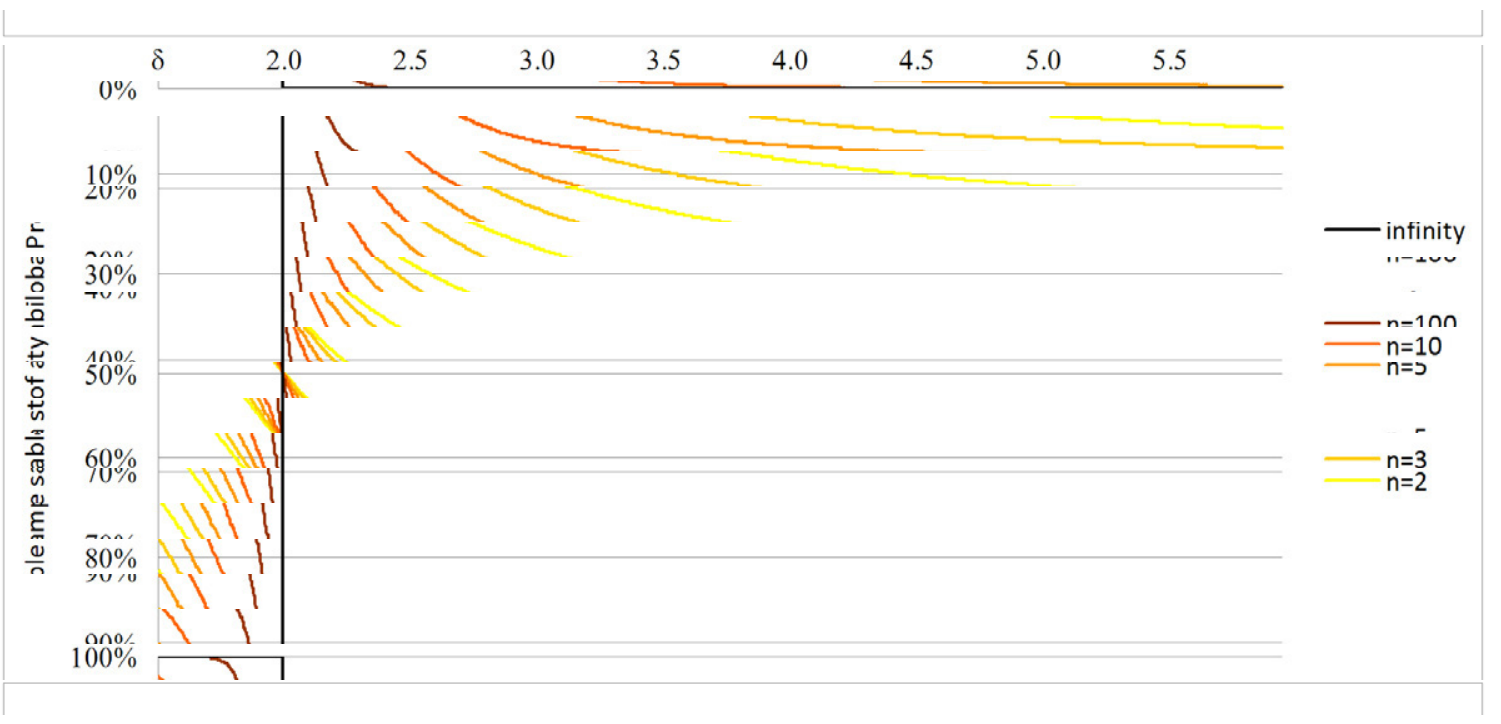




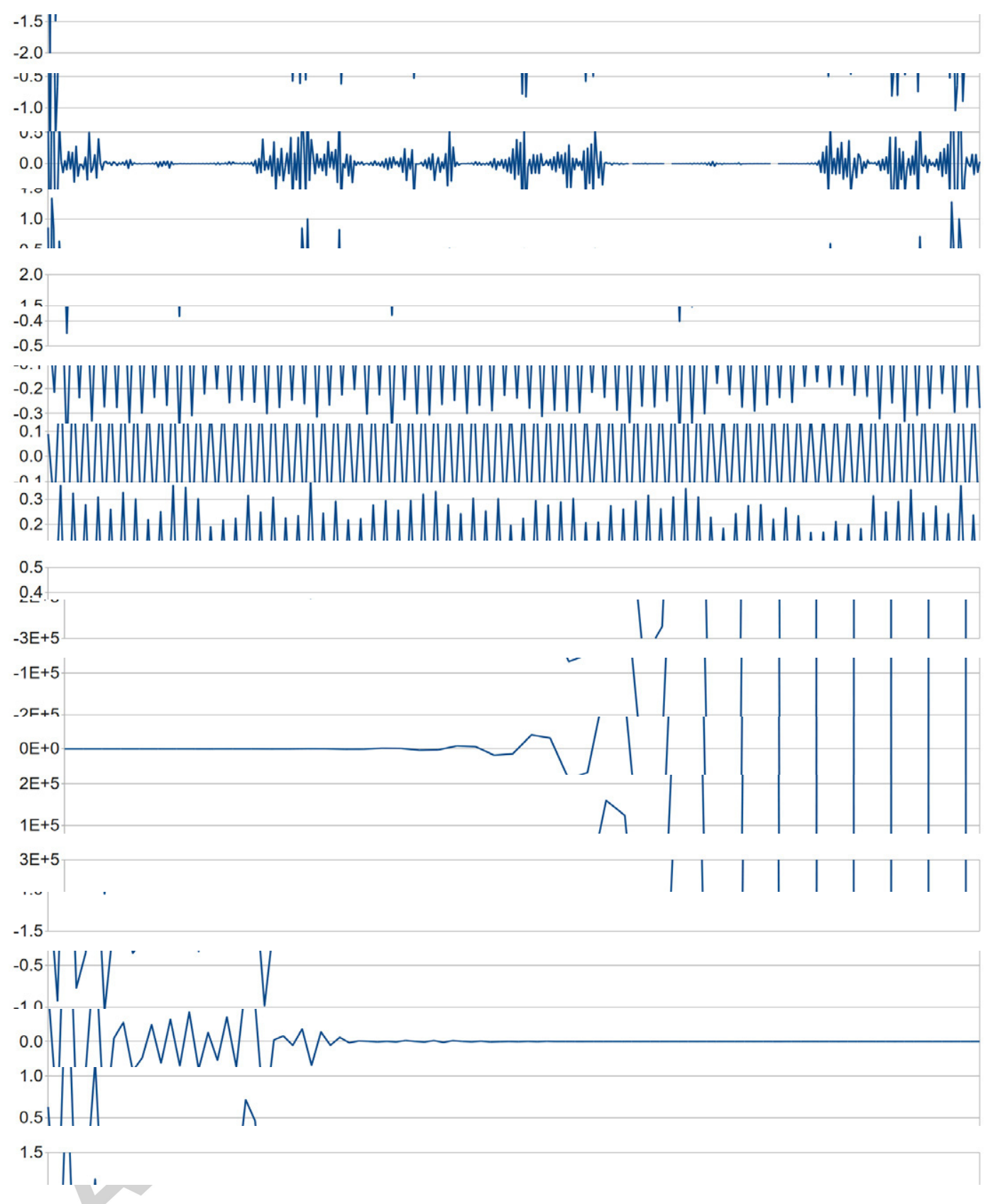

